\title{
Características epidemiológicas de farmacodermias en un hospital privado
}

\author{
Epidemiological characteristics of \\ pharmacodermia in a private hospital
}

Lorena G Moreno Ordaz, * Bianca E López Zenteno, * Enrique J Díaz Greene, ${ }^{\neq}$ Federico L Rodríguez Weber, ${ }^{\S}$ Alejandra Solís Sevilla

Citar como: Moreno OLG, López ZBE, Díaz GEJ, Rodríguez WFL, Solís SA. Características epidemiológicas de farmacodermias en un hospital privado. Acta Med. 2021; 19 (1): 76-80. https://dx.doi.org/10.35366/98574

\section{Resumen}

Introducción: Las farmacodermias representan un problema de salud, con incidencia anual en aumento debido a la automedicación y polifarmacia. Las reacciones cutáneas presentan grados de severidad y a pesar de representar un riesgo sanitario latente, existe poca información en la población mexicana, lo que hace necesaria su caracterización epidemiológica. Material y métodos: Estudio observacional, descriptivo, retrospectivo y transversal. Se incluyeron a pacientes hospitalizados en el Hospital Ángeles Pedregal, mayores de 15 años, con sospecha de efectos adversos a fármacos sistémicos con manifestaciones mucocutáneas, en un periodo comprendido de enero de 2016 a diciembre de 2018. Resultados: Se estudiaron 35,592 individuos, se identificó una incidencia acumulada de 5.28/1,000 pacientes hospitalizados, con predominio del sexo femenino (63\%), edad promedio de 49.1 ( \pm 17$)$ años. El síntoma más frecuente fue prurito, asociado con exantema maculopapular, angioedema, urticaria y choque anafiláctico. Los fármacos involucrados en primer lugar fueron los antibióticos. Conclusiones: Los resultados concuerdan con la literatura internacional, señalando los antibióticos, especialmente las cefalosporinas y fluoroquinolonas, como parte de los fármacos más frecuentemente asociados con farmacodermias. La incidencia de estas manifestaciones alcanzó hasta $0.49 \%$ de la población hospitalizada y uno de cada 62 pacientes padecerán de una reacción medicamentosa que ponga en riesgo la vida.

Palabras clave: Farmacodermias, farmacovigilancia, evento adverso.

\section{Abstract}

Introduction: The pharmacodermias are a health problem with increasing annual incidence, especially due to the growing craze for self-medication and polypharmacy. Drug-associated skin reactions present degrees of severity and despite representing a latent health risk, there is little information on the Mexican population, which makes necessary their epidemiological characterization. Material and methods: Observational, descriptive, retrospective, and cross-sectional study. Hospitalized patients at Hospital Ángeles Pedregal, older than 15 years, with clinical suspicion of adverse effects to systemic drugs with mucocutaneous manifestations, were included in a period from January 2016 to December 2018. Results: 35,592 individuals were studied, a cumulative incidence of 5.28 cases per 1,000 hospitalized patients was identified, most of them female sex $(63 \%)$ with an average age of 49.1 ( \pm 17 ) years. The most frequent symptom was pruritus, associated to maculopapular rash, angioedema, urticaria and anaphylactic shock. With antibiotics identified as the main cause (40.4\%). Conclusions: The results obtained agree with what has been stated internationally pointing to antibiotics, as part of the drugs most frequently associated with the presence of adverse events. The incidence in the presentation of these manifestations reached up to $0.49 \%$ of the hospitalized population and 1 in 62 patients with an adverse event will suffer from a life-threatening drug reaction.

Keywords: Pharmacodermias, pharmacovigilance, adverse event.
* Médico Residente de Medicina Interna. Alumna de la Facultad Mexicana de Medicina de la Universidad La Salle.

₹ Profesor Titular de la Especialidad de Medicina Interna. Facultad Mexicana de Medicina de la Universidad La Salle.

$\S$ Profesor Adjunto de la Especialidad de Medicina Interna. Facultad Mexicana de Medicina de la Universidad La Salle.

^ Área de Farmacovigilancia.

Hospital Ángeles Pedregal. Ciudad de México, México.
Correspondencia:

Lorena G Moreno Ordaz

Correo electrónico: lore.moor23@gmail.com

Aceptado: 09-10-2020.

www.medigraphic.com/actamedica

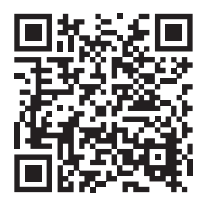




\section{INTRODUCCIÓN}

De acuerdo con la Organización Mundial de la Salud, la reacción adversa a los medicamentos (RAM) es definida como "una respuesta nociva e indeseable que ocurre a dosis normalmente administradas en el ser humano para la profilaxis, el diagnóstico o el tratamiento de una enfermedad, o para la modificación de una función fisiológica".

Las reacciones cutáneas medicamentosas (farmacodermias) se presentan en diversas formas y en ocasiones pueden llegar a ser letales, estas diferencias individuales se atribuyen no sólo a factores ambientales, sino también a enfermedades subyacentes, estilo de vida y factores genéticos. ${ }^{2}$ La piel es un órgano implicado de manera directa en los procesos de farmacocinética y farmacodinamia, razón por la que es capaz de expresar múltiples reacciones clínicas en respuesta a los diferentes estímulos medicamentosos. Del total de reacciones adversas por medicamentos, la piel se afecta aproximadamente en 25 a $30 \%$, que ocurren en $3 \%$ de los pacientes hospitalizados. ${ }^{3}$

Las manifestaciones dermatológicas más frecuentes asociadas con reacciones adversas a los medicamentos son: exantema, urticaria con o sin angioedema, eritema pigmentado fijo, erupción liquenoide, síndrome de Stevens-Johnson, síndrome de Brocq-Lyell y vasculitis. ${ }^{4,5}$

La importancia de las reacciones cutáneas a medicamentos (RCM) se debe a que figuran entre los efectos adversos más comunes, pues representan desde 0.1 hasta $5 \%$, dependiendo de la serie. ${ }^{6,7}$ En México corresponden a $0.67 \%$ de la consulta dermatológica general y hasta $2.9 \%$ de las interconsultas por dermatosis en pacientes hospitalizados.

Existen sistemas nacionales e internacionales que registran esas reacciones adversas; en México el Centro Nacional de Farmacovigilancia, que forma parte de la COFEPRIS desde el año 2001, es el responsable de recibir los informes de la detección de sospechas de reacciones adversas a los medicamentos, vacunas y dispositivos médicos por parte de los profesionales de la salud y laboratorios productores, evaluarlas, valorarlas y retroalimentar la información. ${ }^{8}$

Recientemente, Kim y colaboradores ${ }^{9}$ realizaron una revisión sistemática de reacciones a medicamentos con presencia de eosinofilia y síntomas sistémicos, reportaron que más de $97 \%$ de los pacientes habían experimentado salpullido en la piel e identificaron al hígado como el segundo órgano más afectado. No obstante, esta revisión se enfoca sólo en lo reportado en población menor a 18 años, y a pesar del potencial letal que representan los eventos adversos (EA) medicamentosos, existe poca información actualizada que describa su frecuencia y comportamiento en población adulta mexicana.
Este estudio tuvo el objetivo de analizar y cuantificar los casos de farmacodermias que se detectaron en un periodo de tres años en un hospital de alta especialidad.

\section{MATERIAL Y MÉTODOS}

Se realizó un estudio observacional, retrospectivo y transversal en un periodo comprendido de enero de 2016 a diciembre de 2018. Se seleccionaron expedientes de pacientes mayores de 15 años, con sospecha clínica de efecto adverso a medicamentos, con manifestaciones cutáneas, que se encontraban hospitalizados en los pisos de Medicina Interna, Cirugía y Ginecología-Obstetricia del Hospital Ángeles Pedregal. La población incluida fue el total de casos consecutivos ingresados en el periodo de estudio. La evaluación se realizó por medio del llenado de un formato que permite identificar antecedentes del paciente, fármaco, dosis y vía de administración además de las características clínicas de la reacción adversa para ser clasificada como leve, moderada o grave.

Los datos obtenidos fueron vaciados a una plantilla de Excel y exportados al programa estadístico SPSS versión 25 para su análisis.

\section{RESULTADOS}

Se incluyeron un total de 188 expedientes correspondientes a pacientes que cumplieron con la definición operacional de farmacodermia (manifestación mucocutánea como efecto adverso a fármacos sistémicos) de un total de 35,592 pacientes ingresados al Hospital Ángeles Pedregal. La tasa de incidencia más elevada se presentó durante el periodo enero-diciembre del año 2016 (Figura 1) con 5.89 casos por cada 1,000 pacientes hospitalizados. La incidencia acumulada en el periodo de enero de 2016

Figura 1: Tasa de incidencia a tres años para cada uno de los periodos estudiados.

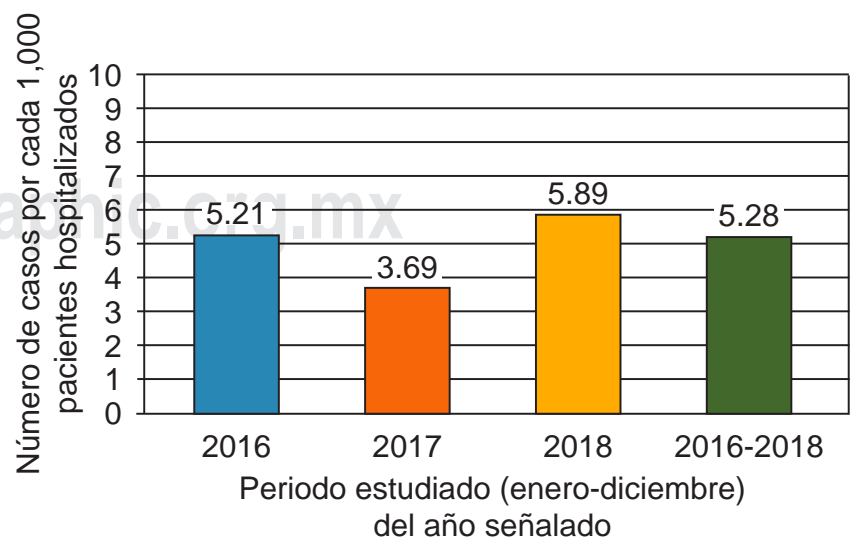


Tabla 1: Características generales de los pacientes con diagnóstico de farmacodermia, periodo 2016-

2018 Hospital Ángeles Pedregal $(\mathrm{N}=188)$.
Variable

Edad (años)

Sexo

Masculino

Femenino

Antecedentes de alergias

Previas a medicamentos

Alimentarias previas

Ambientales

* Media \pm desviación estándar. n (\%)

$49 \pm 17^{*}$

$69(36.7)$

$119(63.3)$

$75(39.8)$

$12(6.3)$

$15(7.9)$ a diciembre de 2018 fue de 5.28 casos por cada 1,000 pacientes hospitalizados.

Del total de la población estudiada, $63 \%$ correspondió al sexo femenino y $36 \%$ al sexo masculino (Tabla 1), con una edad promedio de 49 años. Dentro de los antecedentes, destacó el haber presentado alergia previa a medicamento en $39.8 \%$ de los pacientes incluidos en el estudio.

Respecto a la forma clínica de presentación, el síntoma más frecuente asociado con las reacciones adversas a fármacos fue prurito (Figura 2), los cuales requirieron manejo en unidades de cuidados críticos, como unidades de terapia intensiva o terapia intermedia. De los 188 casos, tres se clasificaron como reacciones graves (una erupción por fármacos con eosinofilia y síntomas sistémicos [DRESS], uno con síndrome de Stevens-Johnson [SSJ] y el último con necrólisis epidérmica tóxica [NET]).

De los fármacos involucrados en los EA de nuestra población, en primer lugar están los antibióticos (40\%), seguidos de medicamentos misceláneos como ranitidina, paracetamol y omeprazol (Figura 3). En lo referente a los antibióticos destaca la utilización de cefalosporinas (34\%), especialmente las cefalosporinas de tercera generación como la ceftriaxona (Tabla 2), seguidas de las fluoroquinolonas (40\%).

Por último, en cuanto al manejo realizado por los médicos de guardia (médicos internos de pregrado y residentes de las especialidades de Medicina Interna, Cirugía y Ginecología-Obstetricia y médicos tratantes), los tratamientos más utilizados incluyeron antihistamínicos anti-H1 en 146 casos (77.6\%), en monotratamiento o aunado a esteroides (principalmente hidrocortisona) en 92 casos (48.9\%), antihistamínicos anti-H2 (ranitidina) 15 casos (7.9\%) y adrenalina en 20 pacientes (10.6\%). Dentro del periodo de estudio no se reportaron desenlaces fatales en ninguno de los casos.

\section{DISCUSIÓN}

Las reacciones medicamentosas son respuestas indeseables secundarias al uso de fármacos prescritos comúnmente, representan las manifestaciones más frecuentes y varían de manera importante en cuanto a clínica y sintomatología; en la actualidad, se consideran un problema de salud pública, ya que representan ingresos hospitalarios, aumento en los días de estancia hospitalaria, aumento de costos intrahospitalarios y pueden repercutir a futuro en la calidad de vida de los pacientes. ${ }^{10}$ La incidencia de farmacodermias reportada en este estudio $(0.05 \%)$ coincide con investigaciones realizadas en años anteriores por Yerenas $C^{11}(0.05 \%)$; sin embargo, hay autores que han referido una incidencia mayor como es el caso de Marzano y $\operatorname{colegas}^{10}(2-3 \%)$ o incluso mucho mayor como Chávez y su equipo, ${ }^{12}$ quienes en un periodo de seis años encontraron 34\% de casos en una Unidad Médica de Alta Especialidad. Estas diferencias pueden deberse a la unidad médica receptora de pacientes, ya que en nuestro estudio se incluyeron pacientes ingresados a un hospital con una gama amplia de especialidades, en cambio, en el caso del estudio realizado por Chávez y su grupo, se incluyeron exclusivamente pacientes asignados al Servicio de Dermatología, por lo que las diferencias en los denominadores no permiten la comparación entre estudios.

Las farmacodermias afectan a todas las razas y tienen predominio por las mujeres $2: 1$, especialmente en edad adulta, ${ }^{10}$ esta situación coincide con lo reportado en nuestra población donde las mujeres representan $63.3 \%$ de los casos descritos. De manera coincidente, un estudio realizado por Watson y colaboradores ${ }^{13}$ reporta una frecuencia de $60 \%$ de EA en el sexo femenino, se hipotetiza que las mujeres corren un mayor riesgo de reacciones adversas,

Figura 2: Características de la sintomatología presentada en los pacientes con farmacodermias $(\mathrm{N}=188)$.

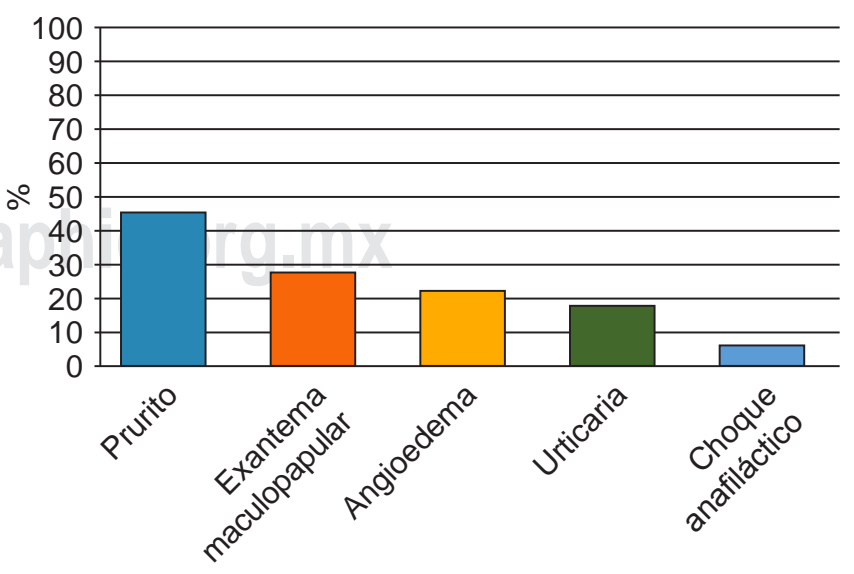


Tabla 2: Antibióticos relacionados con reacciones medicamentosas ( $N=188)$.

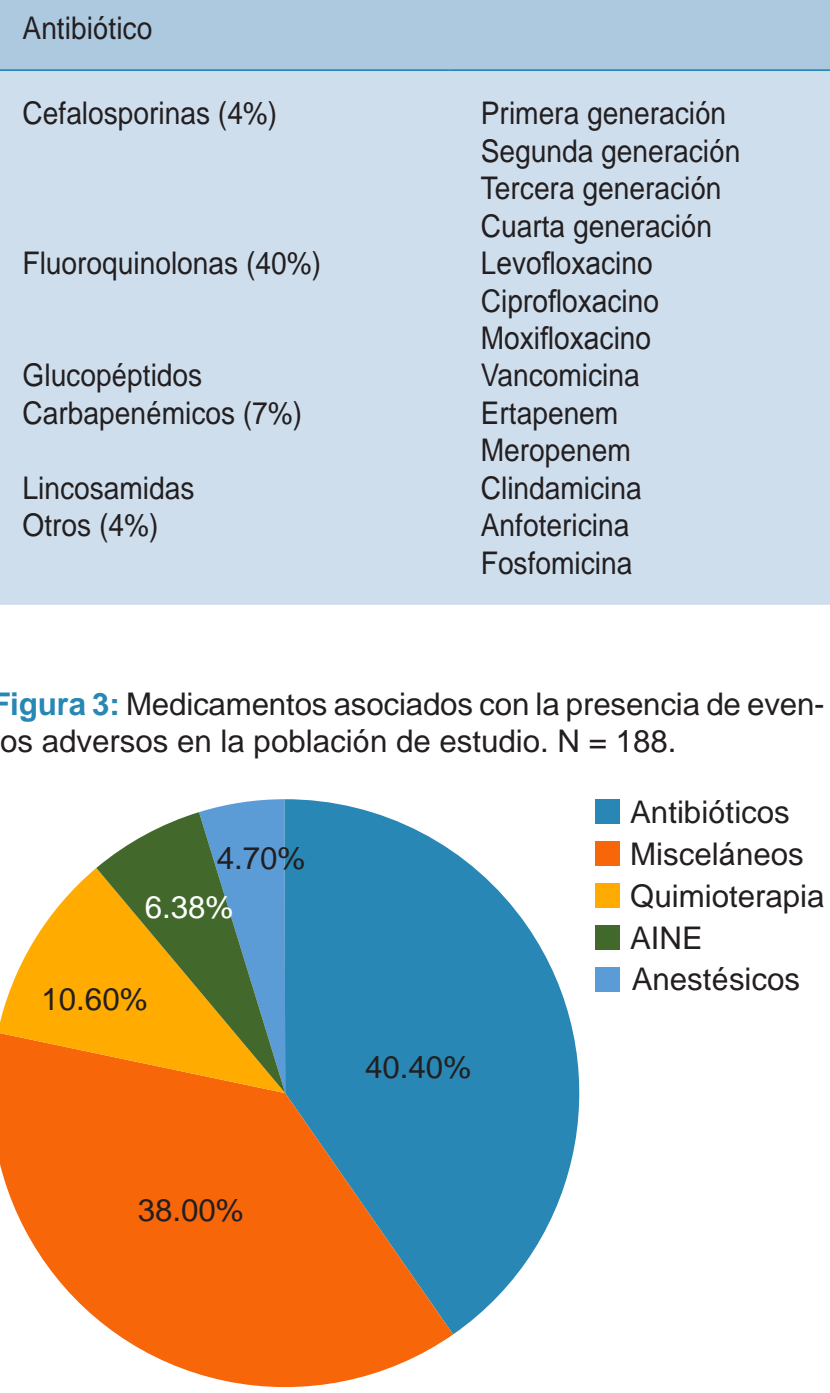

no sólo por la utilización más frecuente de medicamentos, sino también debido a las diferencias entre géneros en los efectos farmacocinéticos y farmacodinámicos y al hecho de estar expuestas a dosis más altas en relación con su peso corporal.

La edad promedio de los casos presentados fue de 49 años, esto concuerda con otros reportes en la literatura, 11,13 en los que la edad promedio de eventos adversos medicamentosos (EAM) se encuentra entre los $48 \pm 7$ años, edad en la que son frecuentes uno o más estados comórbidos, además de la usanza de polifarmacia.

Las manifestaciones presentes en piel pueden ser mediadas por uno de los cuatro tipos de reacciones de hipersensibilidad. La presencia de exantema es el signo más frecuente ante una reacción medicamentosa; sin embargo, existen reacciones cutáneas sistémicas que se consideran graves por poner en riesgo la vida del paciente, esto secundario a la insuficiencia cutánea que pueden producir de manera posterior, entre las más reportadas se encuentra el síndrome de Stevens-Johnson, necrólisis epidérmica tóxica y erupción por fármacos con eosinofilia y síntomas sistémicos, ${ }^{10}$ en nuestra población el síntoma con mayor frecuencia reportado fue prurito y se encontraron sólo tres casos clasificados como reacciones graves (1 DRESS, $1 \mathrm{SSJ}$ y $1 \mathrm{NET}$ ). Desafortunadamente, estas reacciones son imposibles de predecir y requieren la inmediata suspensión o modificación de dosis del fármaco administrado con la futura suspensión permanente del mismo.

Se ha reportado que a nivel mundial la terapia con analgésicos antiinflamatorios no esteroideos (AINEs) representa el primer lugar como detonante de reacciones adversas a medicamentos; ${ }^{14}$ por otra parte, en México el primer lugar como causa de farmacodermias lo ocupan los antibióticos y de éstos, los más frecuentes son el trimetoprim con sulfametoxazol y las penicilinas. En nuestro estudio encontramos que $40 \%$ de los casos fue desarrollado debido a la administración de antibióticos y de éstos las cefalosporinas ocuparon el primer sitio, seguido por el grupo de fluoroquinolonas.

Es importante poner en perspectiva nacional la ocurrencia de los EAM, puesto que se sabe que históricamente existe un subregistro de las mismas por parte del personal médico, ya que en algunos casos se considera necesario sólo notificar las reacciones graves o inesperadas, lo que diluye los datos reales de esta problemática. Se ha descrito que sólo se reporta aproximadamente $18 \%$ de los EAM observados. ${ }^{11}$ 
Se sugiere que, en posteriores estudios, se analice el consumo de otros medicamentos que permitan valorar si el desenlace, o bien la presentación de los eventos, es potenciado debido a alguna interacción medicamentosa. De igual forma, es importante determinar si en la población de estudio fue decisiva la concentración plasmática del fármaco administrado, o bien si la persona que desarrolló el EAM fuese parte de lo que hoy se conoce como metabolizador lento. En la actualidad, se sabe que las enzimas encargadas de metabolizar el fármaco pueden influir mediante la concentración y eliminación del mismo, estas enzimas también pueden tener variaciones genéticas, por ejemplo en poblaciones con CYP2C19*2, la cual es una variante del citocromo P450, se observó que en pacientes con consumo de fenobarbital aumentaba hasta 4.5 veces más el riesgo de presentar una reacción cutánea medicamentosa en comparación con el grupo control; ${ }^{15,16}$ lamentablemente estas variables no pudieron ser medidas en nuestra población de estudio, por lo que se deja la posibilidad, a futuras investigaciones, de incorporar este tipo de enfoques.

\section{CONCLUSIONES}

Este trabajo determina la incidencia, las manifestaciones clínicas y los fármacos involucrados en las reacciones mucocutáneas del Hospital Ángeles Pedregal de la Ciudad de México. A pesar de que es una muestra pequeña, la mayoría de las variables utilizadas coincide con aquellas reportadas en la literatura, por lo que es de gran importancia el continuar estudios de mayor impacto en México con muestras en poblaciones más heterogéneas y de esta forma poder determinar la incidencia nacional.

Ante un abanico grande de posibilidades diagnósticas dermatológicas, sugerimos pensar en el diagnóstico de farmacodermias, sobre todo en pacientes con administración de múltiples fármacos, ya que puede simular otras enfermedades tanto dermatológicas como sistémicas.

\section{REFERENCIAS}

1. Boletín CONAMED-OPS. 2015; 3-5.

2. Aguilar Escobar J, Calvi Fernández R. Toxicodermias. Erupciones cutáneas por fármacos. Gac Med Bol. 2008; 31 (1): 68-68.

3. Lazarou J, Pomeranz BH, Corey PN. Incidence of adverse drug reactions in hospitalized patients: a meta-analysis of prospective studies. JAMA. 1998; 279 (15): 1200-1205.

4. Reynoso DC, Villagrán PM, Rodríguez MN, Rodríguez BS, Acosta RCA, Alcántar LE et al. Abordaje terapéutico del síndrome de necrólisis epidérmica tóxica (síndrome de Brocq-Lyell). Dermatol Rev Mex. 2009; 53 (6): 288-294.

5. Roberts DL, Marks R. Skin reactions to carbamazepine. Arch Dermatol. 1981; 117 (5): 273-275.

6. Sáustegui I, Campos KI, Domínguez J, Méndez S. Reacciones cutáneas adversas a medicamentos. Rev Med Inst Mex Seguro Soc. 2018; 56 (1): 64-70.

7. Hernández SA, Vega ME, Hojyo TMT. Epidemiología de las reacciones cutáneas adversas a fármacos, en el Servicio de Dermatología del Hospital General Dr. Manuel Gea González. Dermatol Rev Mex. 2011; 55 (6): 327-333.

8. NORMA Oficial Mexicana NOM-220-SSA1-2016, Instalación y operación de la farmacovigilancia. Diario Oficial de la Federación. Fecha de publicación: 15 de enero de 2018.

9. Kim GY, Anderson KR, Davis DMR, Hand JL, Tollefson MM. Drug reaction with eosinophilia and systemic symptoms (DRESS) in the pediatric population: A systematic review of the literature. J Am Acad Dermatol. 2020; 83 (5): 1323-1330. doi: 10.1016/j. jaad.2020.03.081.

10. Marzano AV, Borghi A, Cugno M. Adverse drug reactions and organ damage: the skin. Eur J Intern Med. 2016; 28: 17-24.

11. Yerenas García C. Reacciones adversas a fármacos en la Unidad Médica de Alta Especialidad CMNO IMMS. Tesis 2008.

12. Chávez-Lemus FA, Reynoso-von Drateln C, Rodríguez-Martínez N. Prevalencia de las farmacodermias en pacientes hospitalizados en el Servicio de Dermatología de la UMAE del Centro Médico Nacional de Occidente. Periodo 2004-2012. Dermatol Rev Mex. 2014; 58 (4): 339-345.

13. Watson S, Caster O, Rochon PA, den Ruijter H. Reported adverse drug reactions in women and men: Aggregated evidence from globally collected individual case reports during half a century. EClinicalMedicine. 2019; 17: 100188.

14. D'Souza P, Gupta S, Gupta LK, Agarwal N, Akhilesh S. Cutaneous adverse drug reactions. In: Majid I. IADVL recent advances in dermatology. New Delhi, India: Jaypee Brothers Medical Publishers; 2016. pp. 113-129.

15. Rodríguez BL, García VJL, Giral BC, Hernández SD, Jasso GL. Farmacovigilancia III. La experiencia internacional. Rev Med Inst Mex Seguro Soc. 2005; 43 (2): 131-140.

16. Bayry J, Negi VS, Kaveri SV. Intravenous immunoglobulin therapy in rheumatic diseases. Nat Rev Rheumatol. 2011; 7 (6): 349-359. 